Pacific Journal of Mathematics

BARYCENTRIC SUBDIVISIONS 


\section{BARYCENTRIC SUBDIVISIONS}

\section{MARgaret M. Bayer}

A characterization is given of simplicial polytopes that are barycentric subdivisions of regular $\mathrm{CW}$ spheres. It is shown that barycentric subdivisions of connected polyhedral complexes with at least two facets determine the underlying complex uniquely up to duality. Connections with the algorithmic theory of comparability graphs are discussed. The $f$-vectors of regular $\mathbf{C W}$ spheres are characterized.

1. Introduction. Polytopes arise in many areas of mathematics, both pure and applied. Frequently the use of polytopes depends on an understanding of their combinatorial, as opposed to metrical, properties. Much progress has been made in the combinatorial study of polytopes in recent years. The best understood class of polytopes is that of simplicial polytopes. Attempting to use simplicial polytopes to study arbitrary polytopes leads to the study of barycentric subdivisions. This paper studies barycentric subdivisions of geometric cell complexes with a focus on polytopes.

We consider all structures to be in Euclidean space; the definitions are accordingly narrow.

Definition 1 [6]. An incidence polytope is a graded partially ordered set with $\hat{0}$ and $\hat{1}$ satisfying the following properties:

(1) If $C$ and $D$ are maximal chains, then there is a finite sequence of maximal chains, $C=C_{0}, C_{1}, \ldots, C_{k}=D$, where for each $i(0 \leq i \leq k)$ $C_{i}$ contains $C \cap D$, and $C_{i}$ and $C_{i+1}$ differ in exactly one element.

(2) If $x$ and $z$ are elements of the poset with $x<z$ and $\operatorname{rank}(z)-$ $\operatorname{rank}(x)=2$, then there are exactly two elements $y$ such that $x<y<$ $z$.

An incidence polytope is said to have dimension $n$ if the maximal chains contain $n+2$ elements (including $\hat{0}$ and $\hat{1}$ ).

For the next definition we use the following terminology: a set in $\mathbf{R}^{n}$ is an open cell if it is homeomorphic to the interior of the $k$ dimensional unit ball for some $k$; an open cell is regular if its closure is homeomorphic to the closed unit ball. 
Definition 2. A (finite) regular $C W$ complex is a closed subset of $\mathbf{R}^{n}$ that is partitioned into a finite number of regular open cells, each of whose closures is the union of some of these open cells.

Associated with a regular $\mathrm{CW}$ complex $C$ is the poset of the closures of cells, ordered by inclusion, with $\hat{0}=\varnothing$ and $\hat{1}=C$ adjoined. If a regular CW complex is homeomorphic to a sphere it is called a regular CW sphere, and in this case the associated poset is an incidence polytope.

A special type of regular CW sphere is the polytope.

Definition 3. A (convex) polytope is the convex hull of a finite point set in Euclidean space.

A proper face of a polytope is the intersection of the polytope with a supporting hyperplane. The empty set and the polytope itself are called improper faces. The boundary of a polytope (the union of its proper faces) is a regular $\mathrm{CW}$ sphere whose open cells are the interiors of the faces. The associated poset is a lattice, called the face lattice of the polytope.

Definition 4. A polyhedral complex is the union of a finite set of polytopes (faces of the polyhedral complex), the intersection of any two being a face of each.

The most basic type of polytope is the simplex, the convex hull of affinely independent points. A simplicial complex is a polyhedral complex formed as the union of a finite set of simplices. A face of a simplicial complex will often be identified with its vertex set. For a simplicial complex $\Delta$ and face $\sigma$ of $\Delta$, the link of $\sigma$ in $\Delta$ is $\operatorname{link}(\sigma, \Delta)=$ $\{\tau \in \Delta: \sigma \cup \tau \in \Delta$ and $\sigma \cap \tau=\varnothing\}$. A simplicial polytope is a polytope all of whose proper faces are simplices.

Associated with any partially ordered set $P$ is its order complex $\Delta P$. This is a simplicial complex with one vertex for each proper element (element other than 0 or $\hat{1}$ ) of $P$, and a proper face for each chain of proper elements in $P$. The one-skeleton of $\Delta P$ is called the comparability graph of $P$. Comparability graphs have played an important role in graph theory.

A nice characterization of order complexes is known; it is an easy extension of the well-known characterization of comparability graphs by Ghouila-Houri and by Gilmore and Hoffman (see §4). We would like characterizations of order complexes when we restrict the posets to the posets of cells of regular CW complexes (spheres) or to the face lattices of polyhedral complexes (polytopes). In these cases the order complex is known as the barycentric subdivision of the complex. 
In $\S 2$ we characterize order complexes of incidence polytopes. We give a sufficient condition for a combinatorial manifold to be the barycentric subdivision of a regular $\mathrm{CW}$ complex, and describe which simplicial polytopes are barycentric subdivisions of regular $\mathrm{CW}$ spheres. In $\S 3$ we consider uniqueness: if a simplicial complex is the barycentric subdivision of a connected polyhedral complex with at least two facets, then it uniquely determines the underlying complex. Section 4 discusses the use of techniques from the theory of comparability graphs to find the underlying poset of an order complex. The $f$-vectors of regular CW spheres are characterized in $\S 5$.

2. Barycentric subdivisions of regular $\mathrm{CW}$ spheres. We first summarize the situation for incidence polytopes.

A polyhedral complex is called pure if all maximal proper faces (called facets) are of the same dimension. A pure simplicial complex of dimension $n-1$ is called $n$-colorable or completely balanced if its vertices can be given labels, $0,1, \ldots, n$, so that every facet contains one vertex of each label. For a given $n$-coloring of an $(n-1)$-dimensional simplicial complex $\Delta$, a face $F$ of $\Delta$ is called an $S$-face if $S$ is the set of labels on the vertices of $F$. (An $i$-vertex is a vertex with label $i$.)

The order complex of a graded poset has a natural coloring by ranks: each vertex of $\Delta P$ is labeled with the rank of the corresponding element of $P$. If $P$ is graded of rank $n+1$, then $\Delta P$ is a pure, completely balanced simplicial complex of dimension $n-1$.

Definition 5. A $k$-pseudomanifold is a pure $k$-dimensional simplicial complex, such that every $(k-1)$-dimensional face is on exactly two facets and such that given any two facets $F$ and $G$ there is a sequence of facets, $F=F_{0}, F_{1}, \ldots, F_{m}=G$, with $\operatorname{dim}\left(F_{i} \cap F_{i+1}\right)=k-1$ for each $i$. A simplicial complex is a normal pseudomanifold [11] if for every face $\sigma$ (including $\sigma=\varnothing$ ) the link of $\sigma$ in $\Delta$ is a pseudomanifold. (Equivalently, a $k$-pseudomanifold is normal if every face of dimension at most $k-2$ has a connected link.)

Definition 6. An $n$-coloring by $\{0,1, \ldots, n-1\}$ of a simplicial complex $\Delta$ has the double neighbor property if and only if for any face $\sigma$ of $\Delta$, if $\operatorname{dim}(\sigma)=k, 0 \leq i \leq n-1$, and $\sigma$ contains an $(i-1)$-vertex (if $i-1 \geq 0$ ) and an $(i+1$ )-vertex (if $i+1 \leq n-1$ ) but no $i$-vertex, then $\sigma \cup\{x\}$ is a $(k+1)$-dimensional face for exactly two $i$-vertices $x$.

Definition 1 can now be rephrased: A graded poset $P$ is an incidence polytope if and only if its order complex $\Delta P$ with coloring by ranks is a normal pseudomanifold having the double neighbor property. 
Definition 7. A combinatorial $d$-manifold is a $d$-dimensional simplicial complex $\Delta$ such that for any simplex $\sigma, \operatorname{link}(\sigma, \Delta)$ is a PL sphere of $\operatorname{dimension} d-\operatorname{dim}(\sigma)-1$.

THEOREM 1. (i) $\Delta$ is the order complex of an incidence polytope of dimension $n$ if and only if $\Delta$ is an $(n-1)$-dimensional normal pseudomanifold with an $n$-coloring having the double neighbor property.

(ii) If $\Delta$ is an (n-1)-dimensional combinatorial manifold with an $n$ coloring having the double neighbor property, then $\Delta$ is the barycentric subdivision of a regular $C W$ complex.

Proof. (i) We have already observed the "only if" part of (i). We show the converse holds. For a vertex $x$ of $\Delta$, write $\lambda(x)$ for the label on $x$. Define a relation $<$ on the vertices by saying $x_{r}<x_{s}$ if $\left\{x_{r}, x_{s}\right\}$ is an edge of $\Delta$ and $\lambda\left(x_{r}\right)<\lambda\left(x_{s}\right)$. We show $\leq$ is a partial order on the vertices of $\Delta$ by showing transitivity. Assume $x_{i}<x_{j}$ and $x_{j}<x_{k}$, where the subscripts indicate the vertex label. We show $x_{i}<x_{k}$. We know $i<j<k$, so it suffices to show that $\left\{x_{i}, x_{k}\right\}$ is an edge of $\Delta$. Let $F_{1}, F_{2}, \ldots, F_{m}$ be a sequence of facets containing $x_{j}$ such that $x_{i} \in F_{1}, x_{k} \in F_{m}$, and $\operatorname{dim}\left(F_{r} \cap F_{r+1}\right)=n-2$ for all $r$. Let $\sigma$ be the $\{j, j+1, j+2, \ldots, n\}$-face of $F_{m}$; note that $\left\{x_{j}, x_{k}\right\} \subseteq \sigma$. We wish to show that $x_{i} \in \operatorname{link}(\sigma, \Delta)$. (See Figure 1.)

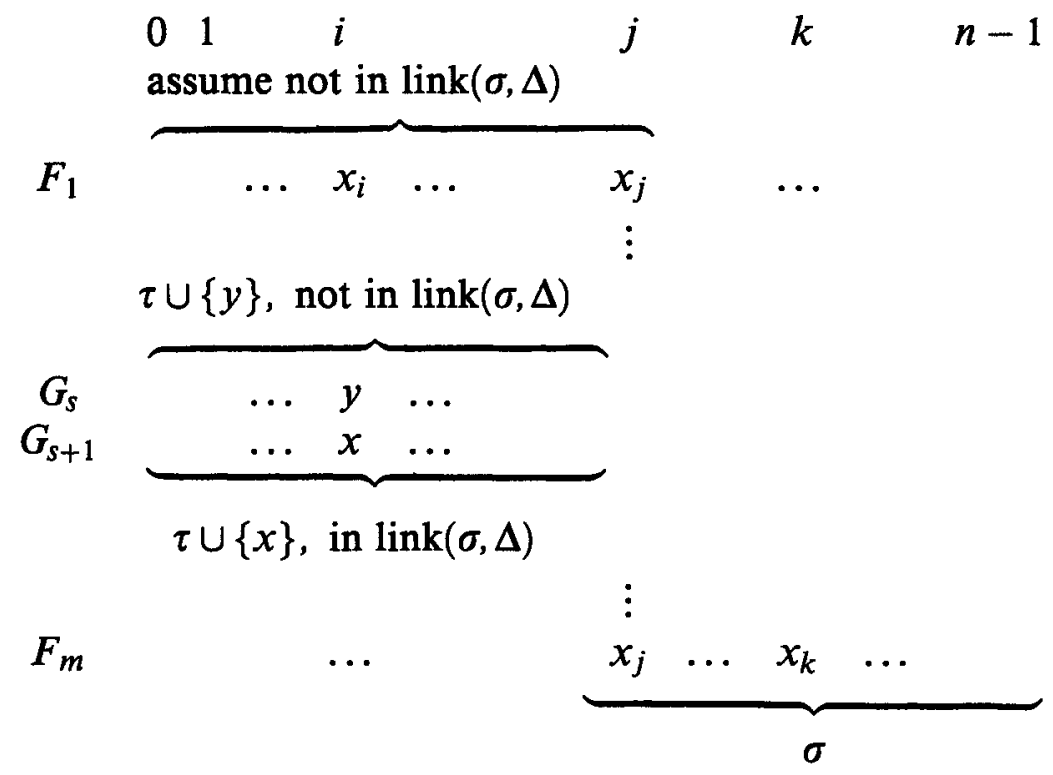

Figure 1

Let $J$ be the subcomplex of $\Delta$ with maximal simplices the $\{0,1, \ldots$, $j-1\}$-faces of $\Delta$. Let $G_{1}, G_{2}, \ldots, G_{t}$ be the maximal subsequence of 
$\left(F_{r} \cap J\right)_{r=1}^{m}$ with no adjacent repetitions. Assume $x_{i} \notin \operatorname{link}(\sigma, \Delta)$. Since $x_{i} \in G_{1}, G_{1}$ is also not in $\operatorname{link}(\sigma, \Delta)$. But $G_{t}$ is in $\operatorname{link}(\sigma, \Delta)$, so there is a greatest index $s$ such that $G_{s}$ is not in $\operatorname{link}(\sigma, \Delta)$. Let $\tau=G_{s} \cap G_{s+1}$, let $y=G_{s} \backslash \tau$, and $q=\lambda(y)<j$. Since $\tau \subseteq G_{s+1} \subseteq \operatorname{link}(\sigma, \Delta), \sigma \cup \tau$ is a face of $\Delta$. So by the double neighbor property there are exactly two $q$-vertices $x$ such that $\sigma \cup \tau \cup\{x\}$ is a face of $\Delta$. So these two vertices with $y$ give three $q$-vertices $w$ such that $\tau \cup\left\{x_{j}, w\right\}$ is a face of $\Delta$. This contradicts the double neighbor property.

Therefore $x_{i} \in \operatorname{link}(\sigma, \Delta)$. But $x_{k} \in \sigma$, so $\left\{x_{i}, x_{k}\right\}$ is an edge of $\Delta$. So we have shown that $<$ is a partial order, and $\Delta=\Delta(P)$, where $P$ is the set of vertices of $\Delta$ with this partial order. The properties of $\Delta(P)$ translate back to $P$ to show that $P$ with $\hat{0}$ and $\hat{1}$ adjoined is an incidence polytope.

(ii) Now suppose $\Delta$ is a combinatorial manifold (with $n$-coloring having the double neighbor property). It is therefore a normal pseudomanifold, so it remains to show that the poset $P$ constructed above is the face poset of a regular CW complex. We use the characterization of these posets, due to Björner [4]. For any $x \in P(\hat{0}, x)$ is the set of elements $y$ of $P$ for which $\hat{0}<y<x$. We get a partially ordered set by restricting $<$ to $(\hat{0}, x) . \Delta(\hat{0}, x)$ is isomorphic to $\operatorname{link}(\sigma, \Delta)$, where $\sigma$ is a face of $\Delta$ corresponding to a saturated chain $x<x_{1}<x_{2}<\cdots<x_{k}$ of $P$. Since $\Delta$ is a combinatorial manifold $\Delta(\hat{0}, x)$ is homeomorphic to a sphere. This is the condition needed for $P$ to be a $C W$ poset. So by [Proposition 3.1, 4] $P$ is isomorphic to the face poset of a regular $\mathrm{CW}$ complex. So $\Delta$ is the barycentric subdivision of a regular $\mathrm{CW}$ complex.

We can ask for an analogous theorem for (normal) pseudomanifolds with boundary. The natural change in hypothesis would be to replace "exactly two" by "at most two" in the double neighbor property. The resulting hypothesis is too weak. As can be seen in the proof of Theorem 1 we need a quite restrictive condition for an $n$-coloring of an $(n-1)$-dimensional normal pseudomanifold $\Delta$ with boundary to be an order complex: for any fixed $\{i-1, i+1\}$-edge $e$ of $\Delta$ there is a constant $c \in\{1,2\}$ such that for any face $F$ containing $e$ but containing no $i$-vertex, there are exactly $c i$-vertices $x$ for which $F \cup\{x\}$ is a face of $\Delta$.

The requirement in Theorem 1 that the pseudomanifold $\Delta$ be normal cannot be dropped. We defer an example until the discussion of uniqueness in the next section. 
For pseudomanifolds the double neighbor property is equivalent to a subset of the generalized Dehn-Sommerville equations. Let $\Delta$ be an $(n-1)$-dimensional simplicial complex with a coloring by $\{0,1, \ldots$, $n-1\}$. Write $f_{S}(\Delta)$ for the number of $S$-faces of $\Delta$, and define the extended $f$-vector of $\Delta$ to be $\left(f_{S}(\Delta)\right)_{S \subseteq\{0,1, \ldots, n-1\}} \in \mathbf{N}^{2^{n}}$. The following equations, known as the generalized Dehn-Sommerville equations, are satisfied by the extended $f$-vectors of the barycentric subdivisions of regular CW spheres (or, more generally, of the order complexes of Eulerian posets):

$$
\sum_{j=i+1}^{k-1}(-1)^{j-i-1} f_{S \cup\{j\}}(P)=\left(1-(-1)^{k-i-1}\right) f_{S}(P)
$$

whenever $S \subseteq\{0,1, \ldots, n-1\},\{i, k\} \subseteq S \cup\{-1, n\}$, and $i<j<k$ implies $j \notin S$.

When $\Delta$ is known to be a pseudomanifold the generalized DehnSommerville equations with $k-i=2$ are equivalent to the double neighbor property. Note also that a certain subset of the generalized Dehn-Sommerville equations (written $h_{S}=h_{\tilde{S}}$ in [1]) hold for the extended $f$-vectors of all $n$-colored $(n-1)$-dimensional spheres. These two subsets of the generalized Dehn-Sommerville equations do not generate all the generalized Dehn-Sommerville equations. However, by Theorem 1 , for $n$-colored $(n-1)$-spheres the double neighbor equations imply the entire set of generalized Dehn-Sommerville equations (see [1] for more on the generalized Dehn-Sommerville equations).

Corollary 2. Let $\Delta$ be a simplicial $n$-dimensional polytope. The following are equivalent.

(1) $\Delta$ has an $n$-coloring having the double neighbor property.

(2) $\Delta$ has an $n$-coloring whose extended $f$-vector satisfies the generalized Dehn-Sommerville equations.

(3) $\Delta$ is the barycentric subdivision of a regular $C W$ sphere of dimension $n-1$.

It is not difficult to show that the barycentric subdivision of any polytope can be realized as the boundary complex of a simplicial polytope (see [9]). So the class of objects described in the corollary includes the barycentric subdivisions of all polytopes. The corollary gives us no idea, however, how to distinguish barycentric subdivisions 
of polytopes within the class of barycentric subdivisions of regular CW spheres. This is an important open question.

3. Uniqueness of barycentric subdivisions. We next consider the question of uniqueness: does a barycentric subdivision determine the underlying regular CW complex? The answer in general is no. This can be seen in a trivial way because the order complexes of any poset and its dual are the same. But this is not all that can go wrong. Below are two face posets of essentially different regular $\mathrm{CW}$ spheres, each having the double bipyramid over a hexagon as its barycentric subdivision.
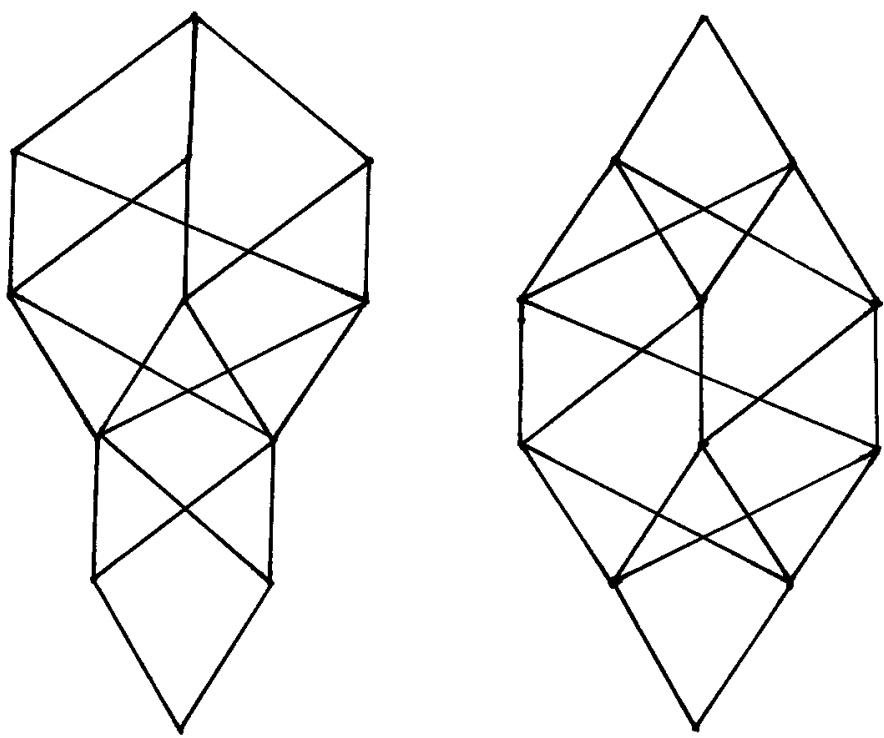

The situation is much better when we restrict ourselves to face lattices of polyhedral complexes.

TheOREM 3. Let $P$ be the face lattice of a connected polyhedral complex with at least two maximal faces, and let $P^{*}$ be its dual poset. If $Q$ is a poset with $\Delta(Q)=\Delta(P)$, then either $Q=P$ or $Q=P^{*}$.

To prove Theorem 3 we need to look at techniques from the theory of comparability graphs (see $[10,12])$.

Let $G$ be a graph with vertex set $V$ and edge set $E$. Let $E_{*}$ be the set of directed edges of $G$, that is, $E_{*}=\{(x, y):\{x, y\} \in E\}$. A directed edge $(x, y)$ is called an orientation of the (undirected) edge $\{x, y\}$; an orientation of $G$ is a function $g: E \rightarrow E_{*}$. The function $g$ 
is called a transitive orientation if $g(E)$ is a transitive relation. (Note that $g$ a function and $g(E)$ transitive imply $g(E)$ is antisymmetric and acyclic.) Clearly, finding a poset of which $G$ is the comparability graph is equivalent to finding a transitive orientation of $G$.

We define an equivalence relation on $E_{*}$. We say that $(u, v)$ directly forces $(w, x)$ if and only if either $u=w$ and $\{v, x\} \notin E$ or $v=x$ and $\{u, w\} \notin E$. Then $(u, v)$ forces $(w, x)$ if and only if there is a sequence of directed edges $(u, v)=\left(u_{0}, v_{0}\right),\left(u_{1}, v_{1}\right), \ldots,\left(u_{k}, v_{k}\right)=(w, x)$ such that for all $i, 0 \leq i \leq k-1,\left(u_{i}, v_{i}\right)$ directly forces $\left(u_{i+1}, v_{i+1}\right)$. This relation is clearly an equivalence relation; the equivalence classes are called implication classes. Furthermore, if $g$ is a transitive orientation of $G$ and $g(E)$ contains one element of an implication class, then $g(E)$ contains the entire implication class. The forcing relation is defined whether $G$ is a comparability graph or not. $G$ is not a comparability graph if and only if some implication class contains both orientations of some edge of $G$.

A graph $G$ is called uniquely partially orderable if and only if it has exactly two transitive orientations, each being the reverse of the other. This is equivalent to the existence of exactly two implication classes, each being the reverse of the other. (The image of each transitive orientation, in this case, is a single implication class.)

Proof of Theorem 3. We show that the one-skeleton of $\Delta(P)$ is uniquely partially orderable. Then any poset having the same order complex (and so the same one-skeleton of the order complex) must be the same as the face lattice of $P$, up to duality.

The proof is by induction on the dimension of $P$. Suppose $P$ is a one-dimensional, connected polyhedral complex with at least two maximal faces. That is, $P$ is a connected graph with at least two edges. Let $G=\Delta(P)$ and label the vertices of $G$ with the dimensions of the corresponding faces of $P$. Note that no edge of $G$ has two vertices of the same label. Choose any edge $e_{0}$ of $G$. If $e$ is any edge of $G$ then by the connectivity of $G$ there is a sequence of edges $e_{0}, e_{1}, \ldots, e_{k}=e$, where for each $i, 0 \leq i \leq k-1, e_{i}$ and $e_{i+1}$ intersect in exactly one vertex, and their noncommon vertices have the same label and thus do not lie on an edge of $G$. So for each $i, 0 \leq i \leq k-1$, an orientation of $e_{i}$ forces an orientation of $e_{i+1}$. Thus there are at most two implication classes, one containing each of the orientations of $e_{0}$. Since we know $\Delta(P)$ has a transitive orientation (given by $P$ ), 
we conclude that there are exactly two implication classes, and they are the reverses of each other.

Now assume that for any connected polyhedral complex $D$ of dimension less than $d$ with at least two maximal faces, the one-skeleton of $\Delta(D)$ is uniquely partially orderable. Let $P$ be a $d$-dimensional connected polyhedral complex having at least two maximal faces. Let $G$ be the one-skeleton of $\Delta(P)$, and label the vertices by the dimensions of the corresponding faces of $P$. Let $P^{\prime}$ be the $(d-1)$-skeleton of $P$, and $G^{\prime}$ the (labeled) one-skeleton of $\Delta\left(P^{\prime}\right)$. The graph $G^{\prime}$ is the subgraph of $G$ induced by all vertices with labels other than $d$. The $(d-1)$-dimensional polyhedral complex $P^{\prime}$ is connected and has at least two maximal faces. So by the induction assumption, $G^{\prime}$ is uniquely partially orderable. It remains to prove that an orientation of $G^{\prime}$ forces an orientation of $G$.

Choose a $d$-dimensional face $F$ of $P$, and let $v_{d}$ be the corresponding vertex of $G$. Since $P$ is connected and has at least two maximal faces, $F$ contains some vertex $\left(v_{0}\right)$ that is on an edge $\left(v_{1}\right)$ not contained in $F$. Thus $\left\{v_{1}, v_{d}\right\}$ is not an edge of $G$, so an orientation of $\left\{v_{0}, v_{1}\right\}$ directly forces an orientation of $\left\{v_{0}, v_{d}\right\}$ (both are edges of $G)$. Now we show that an orientation of $\left\{v_{0}, v_{d}\right\}$ forces an orientation of all other edges containing $v_{d}$. The edges of $G$ containing $v_{d}$ are exactly the edges $\left\{w, v_{d}\right\}$ where $w$ corresponds to a proper face of $F$.

We use the following observation. For any $k$-dimensional face $H$ of $F, 0 \leq k \leq d-2$, there is a $(k+1)$-dimensional face of $F$ not containing $H$. Therefore we can order all proper faces of $F$ so that no two consecutive faces are incident: simply list the faces in order of increasing dimension, making sure that the last $k$-dimensional face is not contained in the first $(k+1)$-dimensional face.

In the corresponding order of the vertices of $\Delta(F)$ no two consecutive vertices lie on an edge. Find such a list starting with $v_{0}: v_{0}=$ $w_{0}, w_{1}, \ldots, w_{s}$. Then for each $i, 0 \leq i \leq s-1$, an orientation of $\left\{w_{i}, v_{d}\right\}$ directly forces an orientation of $\left\{w_{i+1}, v_{d}\right\}$. So an orientation of each edge containing $v_{d}$ is forced by an orientation of $\left\{v_{0}, v_{d}\right\}$, which is itself forced by an orientation of $G^{\prime}$. So an orientation of a single edge of $G^{\prime}$ forces an orientation of all edges of $G$. So $G$ is uniquely partially orderable.

It is clear why the connectedness hypothesis is needed in Theorem 3. What happens if we allow $P$ to have only one maximal face? Then 
$P$ is a (filled in) polytope. Its face lattice looks like

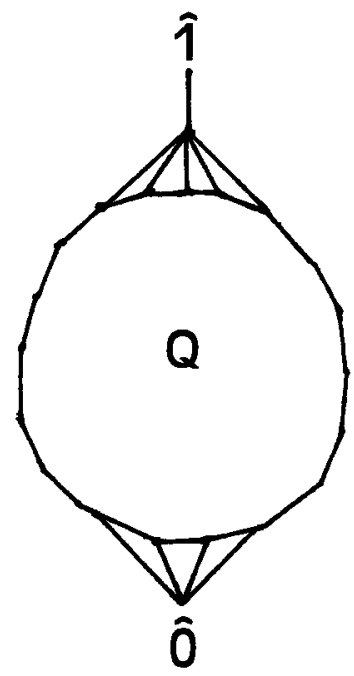

There are three other posets having the same order complex:
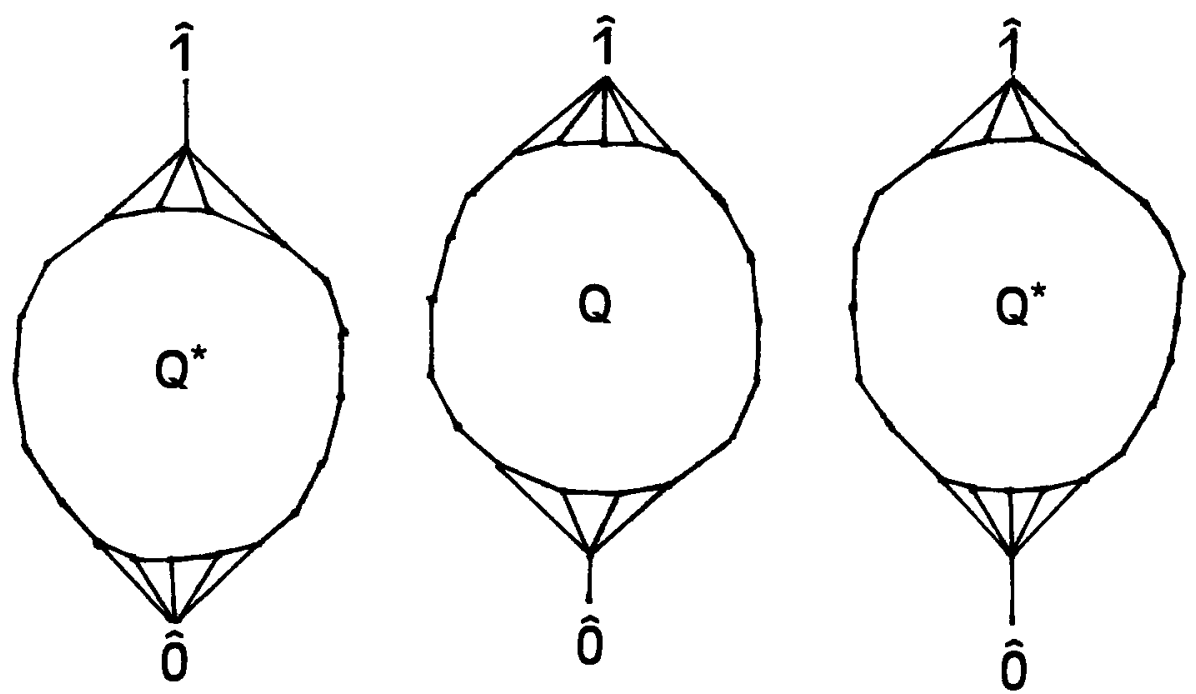

Note that if $Q$ is self-dual then we get four posets, between any two of which there is an inclusion-preserving or inclusion-reversing isomorphism.

We now return to the question of weakening the hypothesis of Theorem 1. We describe here a 5-colored 4-dimensional pseudomanifold, having the double neighbor property, that is not an order complex. Let $P$ be a 5 -dimensional polytope that has two 2 -dimensional faces 
$F$ and $G$ with the property that any facet containing $G$ is disjoint from $F$. Let $\Delta$ be the pseudomanifold obtained by identifying the vertices $v_{F}$ and $v_{G}$ of $\Delta(P)$. The link of $v=v_{F}=v_{G}$ in $\Delta$ is not connected. The identification of the two vertices preserves the double neighbor property of the natural 5-coloring. With this coloring $\Delta$ is not an order complex: the 2-vertex $v$ is adjacent to a 0 -vertex (corresponding to a vertex of $F$ ) and to a 4-vertex (corresponding to a facet containing $G$ ) that are not themselves adjacent. Suppose $\Delta$ has a different 5 -coloring having the double neighbor property. Label $\Delta(P)$ in the corresponding way: $v_{F}$ and $v_{G}$ take the label of $v$, and all other vertices take the label they have in $\Delta$. This coloring of $\Delta(P)$ satisfies the double neighbor property. By Theorem 3 it must be the "dual coloring." In particular the vertex $v$ of $\Delta$ has label 2 , and the same vertices adjacent to $v$ (but with labels switched) prevent $\Delta$ with this coloring from being an order complex.

4. Computational questions. Theorem 1 , with its constructive proof, seems to give a nice method of finding the underlying complex of a barycentric subdivision. It is only practical, however, if we are given a correctly labeled complex $\Delta$. If an $(n-1)$-dimensional manifold is $n$-colorable then it is easy to find an $n$-coloring (see [8] for a simple colorability criterion), and it is unique up to permutation of the labels. We don't want to test $n$ ! label permutations until we hit on one having the double neighbor property. If we restrict ourselves to the case where $\Delta$ is a simplicial polytope, we could perhaps devise a strategy using the full set of generalized Dehn-Sommerville equations to find the correct permutation of the labels. Fortunately, we can instead turn to a known algorithm for comparability graphs.

First observe that the comparability graph of a poset $P$ determines the order complex of $P$. For $C$ is the set of elements of a chain in a poset $P$ if and only if every pair of elements in $C$ is comparable. In the comparability graph $G$ this simply says that the vertex set corresponding to $C$ forms a clique (set of vertices, every pair of which is on an edge). So the order complex $\Delta(P)$ is the simplicial complex with a $k$-simplex for every clique of size $k+1$ in $G$. This gives the following easy characterization of order complexes (observed in [15]).

A missing face of a simplicial complex $\Delta$ is a subset of the vertices that does not form a face of $\Delta$ and that is minimal with respect to this property. 
Proposition 4. A simplicial complex $\Delta$ is the order complex of some poset if and only if the one-skeleton of $\Delta$ is a comparability graph and $\Delta$ has no missing faces of cardinality three or greater.

Note also that if $G$ is the comparability graph of a poset $P$ then any simplicial complex having one-skeleton $G$ is a subcomplex of $\Delta(P)$.

Algorithm 5.2 in [10] determines whether a graph is a comparability graph and, if it is, the algorithm produces a transitive orientation. The algorithm can also determine whether a graph is uniquely partially orderable. Furthermore, the time and space requirements of the algorithm are polynomial in the number of vertices or edges of the graph.

We then have the following method for determining if a simplicial complex $\Delta$ is an order complex. The algorithm is polynomial because comparability graphs are perfect; maximum cliques can be found in linear time ([10]).

(1) Apply the comparability graph algorithm to the one-skeleton $G$ of $\Delta$.

(2) If $G$ is not a comparability graph, then $\Delta$ is not an order complex.

(3) If $G$ is a comparability graph, compute the number of cliques of $G$ and compare with the number of faces of $\Delta$. If they are unequal then $\Delta$ is not an order complex.

(4) If $G$ is a comparability graph with the same number of cliques as faces of $\Delta$, then $\Delta$ is the order complex of any poset with comparability graph $G$.

(5) If $G$ is not uniquely partially orderable, then $\Delta$ is an order complex, but it is not the barycentric subdivision of a connected polyhedral complex with at least two maximal faces.

5. $f$-vectors of regular $\mathrm{CW}$ spheres. A main open question in the study of polytopes is how many faces of each dimension polytopes may have. For a regular $\mathrm{CW}$ complex $P$ let $f_{i}$ be the number of $i$ dimensional cells (or faces), with the convention that $f_{-1}=1$. The $f$-vector of $P$ is then $f(P)=\left(f_{0}, f_{1}, \ldots, f_{n}\right)$. We are far from a characterization of $f$-vectors of polytopes. A characterization of the $f$ vectors of simplicial polytopes was conjectured by McMullen [13] and proved by Stanley [16] and Billera and Lee [2]. Björner and Kalai [5] characterized the $f$-vectors that could be realized by simplicial complexes or regular CW complexes with given homology ranks. The following theorem is related. 
Theorem 5. Let $f=\left(f_{0}, f_{1}, \ldots, f_{n}\right) \in \mathbf{N}^{n+1}$. The following are equivalent:

(i) $f$ is the $f$-vector of a regular $C W$ sphere of dimension $n$;

(ii) $f$ is the $f$-vector of a regular $C W$ sphere of dimension $n$ having polytopal barycentric subdivision; 1 .

(iii) $\sum_{i=-1}^{n}(-1)^{n-i} f_{i}=1$ and, for $0 \leq j \leq n-1, \sum_{i=-1}^{j}(-1)^{j-i} f_{i} \geq$

Proof. We show that (i) implies (iii) and that (iii) implies (ii). Assume $f$ is the $f$-vector of a regular CW sphere $P$ of dimension $n$. For any $j, 0 \leq j \leq n$, the sum $\sum_{i=-1}^{n}(-1)^{n-i} f_{i}$ is the rank of the $j$ th homology group of the $j$-skeleton of $P$. For $j=n$ this rank is 1 . For $j<n$ this rank is positive, because $P$ has some $(j+1)$-dimensional cell, and the sum of the $j$-dimensional cells in its boundary is a nonzero cycle.

We now prove (iii) implies (ii) by induction on $n$. If $n=0$ the only vector satisfying (iii) is (2). This is the $f$-vector of the boundary of an interval, which is its own barycentric subdivision. So assume (iii) implies (ii) for vectors $\left(f_{0}, f_{1}, \ldots, f_{n-1}\right)$. Suppose the vector $f=$ $\left(f_{0}, f_{1}, \ldots, f_{n}\right) \in \mathbf{N}^{n+1}$ satisfies $\sum_{i=-1}^{n}(-1)^{n-i} f_{i}=1$ and, for $0 \leq j \leq$ $n-1, \sum_{i=-1}^{j}(-1)^{j-i} f_{i} \geq 1$. Let

$f_{n-1}^{\prime}=\sum_{i=-1}^{n-2}(-1)^{n-2-i} f_{i}+1 \geq 2, \quad$ and let $f^{\prime}=\left(f_{0}, f_{1}, \ldots, f_{n-2}, f_{n-1}^{\prime}\right)$.

Then $f^{\prime}$ satisfies (iii), so by the induction assumption there is a regular CW sphere $P^{\prime}$ of dimension $n-1$ having polytopal barycentric subdivision. Adjoin two $n$-cells to $P^{\prime}$ to form an $n$-sphere with $f$-vector $\left(f_{0}, f_{1}, \ldots, f_{n-1}^{\prime}, 2\right)$. The barycentric subdivision of this sphere is a bipyramid over the barycentric subdivision of $P^{\prime}$, and hence can be realized as a polytope.

Now repeat the following operation $f_{n}-2$ times, adjoining $f_{n}-2$ $(n-1)$-cells and $f_{n}-2 n$-cells. Choose an $(n-1)$-cell $C$ of the regular $\mathrm{CW}$ sphere. It is contained in the closures of two $n$-cells $D_{1}$ and $D_{2}$. Form a new regular $\mathrm{CW}$ sphere by replacing $C$ with two copies of $C$ surrounding a new $n$-cell, one copy of $C$ contained in the closure of each of $D_{1}$ and $D_{2}$. The effect in the barycentric subdivision is to perform stellar subdivisions on the two edges joining the vertex corresponding to $C$ with those corresponding to the $n$-cells $D_{i}$. The result of a stellar subdivision can be realized as a polytope. Since 
$f_{n-1}^{\prime}+f_{n}-2=f_{n-1}$, we have constructed a regular $\mathrm{CW}$ sphere with $f$-vector $\left(f_{0}, f_{1}, \ldots, f_{n}\right)$ and with polytopal barycentric subdivision. $\square$

Observe that in each dimension there is a unique least $f$-vector of a regular CW sphere: $(2,2, \ldots, 2)$. A regular $(n-1)$-dimensional $\mathrm{CW}$ sphere having two cells of each dimension has as its barycentric subdivision the $n$-dimensional crosspolytope. This is the minimal $n$ colorable simplicial sphere (for more on colorable complexes see [3]).

The equivalence of (i) and (ii) in Theorem 5 says that spheres with polytopal barycentric subdivisions cannot be identified by their $f$ vectors. (We do not know an example of a regular $\mathrm{CW}$ sphere whose barycentric subdivision is not realizable as a polytope.) A similar question for simplicial spheres (not necessarily barycentric subdivisions) is open-it is not known whether the set of $f$-vectors of simplicial spheres is the same as the set of $f$-vectors of simplicial polytopes.

6. Related work and open questions. Dress [7] studied barycentric subdivisions from a slightly different perspective. Consider the Coxeter group

$$
\begin{aligned}
\Sigma=\left\langle\sigma_{0}, \sigma_{1}, \ldots, \sigma_{n-1}\right| \sigma_{i}^{2} & =\left(\sigma_{i} \sigma_{k}\right)^{2}=1 \\
& \text { for } i, k=0,1, \ldots, n-1 \text { and }|i-k| \geq 2\rangle .
\end{aligned}
$$

For an $n$-colored ( $n-1)$-dimensional pseudomanifold $\Delta$, and a facet $F$ of $\Delta$, define $\sigma_{i}(F)$ to be the facet of $\Delta$ differing from $F$ only in the $i$-vertex. If $\Delta$ is the order complex of a poset, this defines a $\Sigma$-action on the facets of $\Delta$. In a vein similar to this paper, Dress considered the reverse question. He determined under what conditions a set $\Omega$, having a $\Sigma$-action, is isomorphic (as a $\Sigma$-set) to the set of facets of a pseudomanifold that is the order complex of some poset. He further studied the relationship between the action of a group on a poset and the action of the group on the facets of the order complex.

Besides the characterization of face posets of regular $\mathrm{CW}$ complexes, several interesting and related topics are covered in [4]. There Björner discusses shellability of regular $\mathrm{CW}$ complexes and the realization as regular CW posets of certain partial orderings, such as Bruhat order and the poset of intervals of the face lattice of a polytope.

Many open problems remain. The greatest of these is to characterize barycentric subdivisions of polytopes (among barycentric subdivisions of regular $\mathrm{CW}$ spheres). A characterization of the extended $f$-vectors 
of (barycentric subdivisions of) regular $\mathrm{CW}$ spheres would also be desirable.

The class of incidence polytopes is one generalization of the face posets of regular CW spheres. Another is the class of Eulerian posets (see [17] for the definition); for these posets the generalized DehnSommerville equations hold [1]. We would like a characterization of barycentric subdivisions of Eulerian posets-it is not enough for a simplicial complex to have an $n$-coloring satisfying the generalized Dehn-Sommerville equations.

The author would like to thank Anders Björner for discussion helpful to this work.

\section{REFERENCES}

[1] M. M. Bayer and L. J. Billera, Generalized Dehn-Sommerville relations for polytopes, spheres and Eulerian partially ordered sets, Invent. Math., 79 (1985), 143-157.

[2] L. J. Billera and C. W. Lee, A proof of the sufficiency of McMullen's conditions for $f$-vectors of simplicial polytopes, J. Combinatorial Theory Ser. A, 31 (1981), 237-255.

[3] L. J. Billera and K. E. Magurn, Balanced subdivision and enumeration in balanced spheres, Disc. and Computational Geometry, 2 (1987), 297-317.

[4] A. Björner, Posets, regular CW complexes and Bruhat order, European J. Combin., 5 (1984), 7-16.

[5] A. Björner and G. Kalai, On $f$-vectors and homology, preprint.

[6] L. Danzer and E. Schulte, Reguläre Inzidenzkomplexe, I, Geom. Dedicata, 13 (1982), 295-308.

[7] A. W. M. Dress, Regular polytopes and equivariant tessellations from a combinatorial point of view, in L. Smith, ed., Algebraic Topology, Göttingen 1984, Lecture Notes in Mathematics 1172, Springer-Verlag, New York, 1985, 56-72.

[8] R. Edwards, An amusing reformulation of the four color problem, Notices Amer. Math. Soc., 24 (1977), A257-A258.

[9] G. Ewald and G. C. Shephard, Stellar subdivisions of boundary complexes of convex polytopes, Math. Ann., 210 (1974), 7-16.

[10] M. C. Golumbic, Algorithmic Graph Theory and Perfect Graphs, Academic Press, New York, 1980.

[11] G. Kalai, Rigidity and the lower bound Theorem I, Invent. Math., 88 (1987), 125-151.

[12] D. Kelly, Comparability graphs, in I. Rival, ed., Graphs and Orders, D. Reidel Publishing Company, 1985, 3-40.

[13] P. McMullen and G. C. Shephard, Convex Polytopes and the Upper Bound Conjecture, Cambridge University Press, Cambridge, 1971.

[14] E. Schulte, Regular incidence-polytopes with Euclidean or toroidal faces and vertex-figures, J. Combinatorial Theory Ser. A, 40 (1985), 305-330. 
[15] R. Stanley, Balanced Cohen-Macaulay complexes, Trans. Amer. Math. Soc., 249 (1979), 139-157.

[16] - The number of faces of a simplicial convex polytope, Adv. in Math., 35 (1980), 236-238.

[17] _ Some aspects of groups acting on finite posets, J. Combinatorial Theory Ser. A, 32 (1982), 132-161.

Received August 10, 1987. The author was supported in part by a National Science Foundation grant while at Northeastern University.

UNIVERSITY OF KANSAS

LAWRENCE, KS 66045 


\section{PACIFIC JOURNAL OF MATHEMATICS}

\section{EDITORS}

\author{
V. S. VARADARAJAN \\ (Managing Editor) \\ University of California \\ Los Angeles, CA 90024 \\ HeRBERT ClEMENS \\ University of Utah \\ Salt Lake City, UT 84112 \\ R. FINN \\ Stanford University \\ Stanford, CA 94305
}

\section{HERMANN FLASCHKA \\ University of Arizona \\ Tucson, AZ 85721}

VAUghan F. R. Jones

University of California

Berkeley, CA 94720

ROBION KIRBY

University of California

Berkeley, CA 94720

\author{
C. C. MOORE \\ University of California \\ Berkeley, CA 94720
}

HAROLD STARK

University of California, San Diego

La Jolla, CA 92093

\section{ASSOCIATE EDITORS}
R. ARENS
E. F. BECKENBACH
B. H. NEUMANN
F. WOLF
K. YOSHIDA
(1906-1982)

\section{SUPPORTING INSTITUTIONS}

\begin{abstract}
UNIVERSITY OF ARIZONA
UNIVERSITY OF BRITISH COLUMBIA

UNIVERSITY OF CALIFORNIA

MONTANA STATE UNIVERSITY

UNIVERSITY OF NEVADA, RENO

NEW MEXICO STATE UNIVERSITY

OREGON STATE UNIVERSITY
\end{abstract}
CALIFORNIA INSTITUTE OF TECHNOLOGY

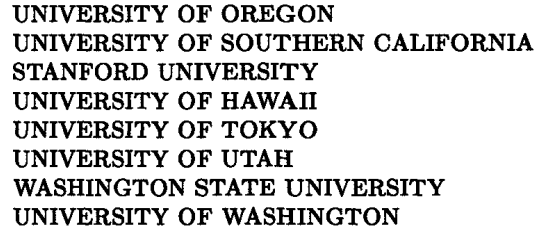

The Supporting Institutions listed above contribute to the cost of publication of this Journal, but they are not owners or publishers and have no responsibility for its content or policies.

Mathematical papers intended for publication in the Pacific Journal of Mathematics should be in typed form or offset-reproduced (not dittoed), double spaced with large margins. Please do not use built up fractions in the text of the manuscript. However, you may use them in the displayed equations. Underline Greek letters in red, German in green, and script in blue. The first paragraph must be capable of being used separately as a synopsis of the entire paper. In particular it should contain no bibliographic references. Please propose a heading for the odd numbered pages of less than 35 characters. Manuscripts, in triplicate, may be sent to any one of the editors. Please classify according to the scheme of Math. Reviews, Index to Vol. 39. Supply name and address of author to whom proofs should be sent. All other communications should be addressed to the managing editor, or Elaine Barth, University of California, Los Angeles, California 90024.

There are page-charges associated with articles appearing in the Pacific Journal of Mathematics. These charges are expected to be paid by the author's University, Government Agency or Company. If the author or authors do not have access to such Institutional support these charges are waived. Single authors will receive $\mathbf{5 0}$ free reprints; joint authors will receive a total of 100 free reprints. Additional copies may be obtained at cost in multiples of 50 .

The Pacific Journal of Mathematics is issued monthly as of January 1966. Regular subscription rate: $\$ 190.00$ a year (5 Vols., 10 issues). Special rate: $\$ 95.00$ a year to individual members of supporting institutions.

Subscriptions, orders for numbers issued in the last three calendar years, and changes of address should be sent to Pacific Journal of Mathematics, P.O. Box 969, Carmel Valley, CA 93924, U.S.A. Old back numbers obtainable from Kraus Periodicals Co., Route 100, Millwood, NY 10546.

The Pacific Journal of Mathematics at P.O. Box 969, Carmel Valley, CA 93924 (ISSN 0030-8730) publishes 5 volumes per year. Application to mail at Second-class postage rates is pending at Carmel Valley, California, and additional mailing offices. Postmaster: send address changes to Pacific Journal of Mathematics, P.O. Box 969, Carmel Valley, CA 93924.

PUBLISHED BY PACIFIC JOURNAL OF MATHEMATICS, A NON-PROFIT CORPORATION Copyright (C) 1988 by Pacific Journal of Mathematics 


\section{Pacific Journal of Mathematics}

Vol. 135, No. $1 \quad$ September, 1988

Margaret M. Bayer, Barycentric subdivisions $\ldots \ldots \ldots \ldots \ldots \ldots \ldots \ldots \ldots$

Eung Chun Cho, $s$-Smith equivalent representations of dihedral groups . . . . 17

Avner Friedman and Jindrich Necas, Systems of nonlinear wave equations

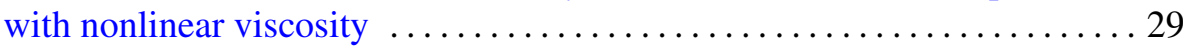

Kyong Taik Hahn, Nontangential limit theorems for normal mappings . . . . 557

Eloise A. Hamann, Evan Green Houston, Jr. and Jon Lee Johnson,

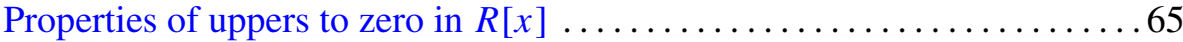

José M. Souto Menéndez, On a theorem due to Cassels ............. 81

Courtney Hughes Moen, Irreducibility of unitary principal series for

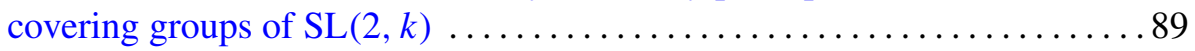

Frank M. Neubrander, Integrated semigroups and their applications to the

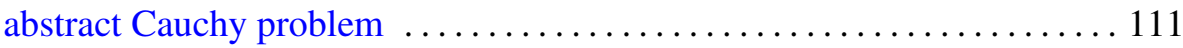

C. K. Qu and Roderick Sue-Chuen Wong, Szegő's conjecture on Lebesgue

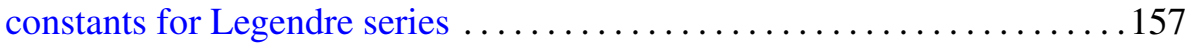

John Brendan Sullivan, The Euler character and cancellation theorems for

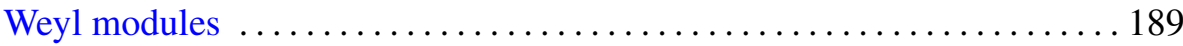

